\title{
Coruscations and Requirements for Quality of Services in Mobile Ad hoc Networks
}

\author{
Mukesh Kumar \\ Department of Computer \\ Engineering \\ The Technological Institute of Textile \\ and Science, Bhiwani-127021, \\ Haryana - India
}

\author{
Rahul Rishi \\ Associate Professor \\ Department of Computer Engineering \\ The Technological Institute of Textile \\ and Science, Bhiwani-127021, \\ Haryana - India
}

\author{
D.K.Madan \\ Associate Professor \\ Department of Mathematics \\ The Technological Institute of \\ Textile and Science
}

Bhiwani-127021, Haryana - India

\begin{abstract}
A mobile Ad hoc network (MANET) is an autonomous system of mobile routers (and associated hosts) connected by wireless links. In some applications, the routers are fixed and sometimes mobile free to move randomly and organize themselves arbitrarily. Due to mobile nature, the network's wireless topology may change rapidly and unpredictably. Such a network may operate in a stand-alone fashion, or may be connected to the larger Internet. MANET'S were initially proposed for use in military and battle field, due to the rapid expansion of the Multimedia Technology, Mobile Technology and civilian applications has to strictly adhere to QoS. The paper presents the description about the QoS and the issues of MANETS like Routing, Medium (or Channel) access, Mobility Management, Security and Reliability, and Power Consumption and also the current approaches including models and solution strategies. The paper presents a literature study on the requirements of Quality of Services (QoS) in Mobile Ad-Hoc Networks (MANET'S) which is universally growing area.
\end{abstract}

Keywords: - QoS, MANET, WLAN, MAC, RSVP

\section{INTRODUCTION}

A mobile ad-hoc network (MANET) is a kind of wireless ad-hoc network, and is a self-configuring network of mobile routers connected by wireless links. The routers are free to move randomly so the network's wireless topology may change rapidly and unpredictably. MANETs are useful in many applications because they do not need any infrastructure support. Collaborative computing and communications in smaller areas (building organizations, conferences, etc.) can be set up using MANETS. Communications in battlefields and disaster recovery areas are further examples of application environments.

Quality of Service (QoS) based routing is defined in RFC 2386 [8] as a "Routing mechanism under which paths for flows are determined based on some knowledge of resource availability in the network as well as the QoS requirement of flows."

QoS is usually defined as a set of service requirements that needs to be met by the network while transporting a packet stream from a source to its destination. The network needs are governed by the service requirements of end user applications. The network is expected to guarantee a set of measurable pre-specified service attributes to the users in terms of end-to-end performance, such as delay, bandwidth, probability of packet loss, delay variance (jitter), etc. Power consumption is another QoS attribute which is more specific to MANETs.

QoS support in MANETs spans over all the layers in the network: QoS models specify an architecture in which some kinds of services could be provided. It is the system goal that has to be implemented.

QoS Adaptation hides all environment-related features from awareness of the multimedia-application above and provides an interface for applications to interact with QoS control.

QoS signaling acts as a control center in QoS support. The functionality of QoS signaling is determined by the QoS model.

QoS routing is part of the network layer and searches for a path with enough resources but does not reserve resources.

QoS MAC protocols are essential components in QoS for MANETs. QoS supporting components at upper layers, such as QoS signaling or QoS routing assume the existence of a MAC protocol, which solves the problems of medium contention, supports reliable communication, and provides resource reservation.

This paper is divided into sections. The section 2 discusses the issues and difficulties related to MANET. Section 3 shows the quality of services related issues and the architecture of QoS is discusses in section 4. Section 5 QoS model classified into three categories and section 6 QoS technologies which are used.

\section{ISSUES AND DIFFICULTIES IN MANETS}

MANETs differ from the traditional wired Internet infrastructures. The differences introduce difficulties for achieving Quality of Service in such networks. Some of the problems as listed below:

Dynamic topologies: Nodes are free to move arbitrarily; thus, the network topology - which is typically multi-hop - may change randomly and rapidly at unpredictable times, and may consist of both bidirectional and unidirectional links.

Bandwidth-constrained, variable capacity links: Wireless links will continue to have significantly lower capacity than their hardwired counterparts. In addition, the realized throughput of wireless communications - after accounting for the effects of multiple access, fading, noise, and interference conditions, etc.- is often 
much less than a radio's maximum transmission rate. One effect of the relatively low to moderate link capacities is that congestion is typically the norm rather than the exception, i.e. aggregate application demand will likely approach or exceed network capacity frequently. As the mobile network is often simply an extension of the fixed network infrastructure, mobile ad hoc users will demand similar services. These demands will continue to increase as multimedia computing and collaborative networking applications rise.

Energy-constrained operation: Some or all of the nodes in a MANET may rely on batteries or other exhaustible means for their energy. For these nodes, the most important system design criteria for optimization may be energy conservation.

\section{QUALITY OF SERVICE}

In other words, Quality of Service (QoS) refers to a set of service requirements that needs to be met by the network while transporting a packet stream from a source to its destination. Informally, it refers to the probability of a packet passing between two points in the network. The network is expected to guarantee a set of measurable pre-specified service attributes to the users in terms of end-to-end performance, such as delay, bandwidth, probability of packet loss, delay variance (jitter), power consumption etc. The challenges of supporting QoS in ad hoc networks are how to reserve bandwidth and how to guarantee the specified delay for real-time application data flows. For wireless transmissions, the channel is shared among neighbors. Therefore, the available bandwidth depends on the neighboring traffic status, as does the delay. Due to this characteristic, supporting QoS cannot be done by the host itself, but cooperation from the hosts within a node's interference range is needed. This requires an innovative design to coordinate the communication among the neighbors in order to support QoS in MANETs. Furthermore, the distributed organization of MANETs brings additional challenges to collaboration for supporting QoS[1,5].

The wireless communication was originally developed for army use, because of its ease of mobility, installation and flexibility; later on it was made available to civilian use also. With the increasing demand and penetration of wireless services, users of wireless network now expect quality of service and performance comparable to what is available from fixed networks. Some of factors that influence QoS of Wireless Network include:

a) Throughput of Network

Represents the total number of bits (in bits/sec) forwarded from wireless LAN layers to higher layers in all WLAN nodes of the network.

b) Retransmission Attempts

Total number of retransmission attempts by all WLAN MACs in the network until either packet is successfully transmitted or it is discarded as a result of reaching short or long retry limit.

c) Data Dropped

Data dropped due to unavailability of access to medium.

d) Medium Access Delay

It includes total of queuing and contention delays of the data.

\section{QUALITY OF SERVICE ARCHITECTURE}

The QoS-A is a layered architecture of services and mechanisms for QoS management and control of continuous media flows in multiservice networks. The most fundamental architectural concept is the notion of a flow. A flow characterises the production, transmission and eventual consumption of a single media stream as an integrated activity governed by a single statement of QoS. Flows are always simplex but can be either unicast or multicast. They may carry a range of data types including both continuous media and control data such as messages or RPC packets. The realisation of the flow concept demands active QoS management and tight integration between the device management thread scheduling, communications protocol and network components of the end-toend data path.

In functional terms, the QoS- A illustrated in Figure 1 is broadly divided into a number of layers and planes. The upper layer consists of a distributed applications platform augmented with services to provide multimedia communications and QOS configuration in an object-based environment [1]. Below the platform level is an orchestration layer which provides multimedia synchronisation services across multiple related application flows and jitter correction [2]. Supporting this is a transport layer which contains a range of QoS configurable protocols. For example, separate protocols are provided for continuous media and constrained latency message protocols [3].

The vertical planes in the QoS-A, of which there are three, are as follows:

\section{i) The protocol plane}

This consists of a user plane and a control plane. Control generally requires a low latency full duplex assured service whereas multimedia data generally requires a range of non-assured, high throughput simplex services.

\section{ii) The QoS maintenance plane}

The QoS maintenance plane contains a number of layer specific QoS managers. These are each responsible for the fine grained monitoring and maintenance of their associated protocol entities. Based on flow monitoring information and a user supplied service contract, QoS managers maintain the level of QoS in the managed flow by means of fine grained resource tuning strategies.

\section{iii) The flow management plane}

This is responsible for flow establishment (including flow admission control, resource reservation and QoS based routing), QoS re-negotiation, QoS mapping (which translates QoS representations between layers) and QoS adaptation (which implements coarse grained QoS maintenance control). 


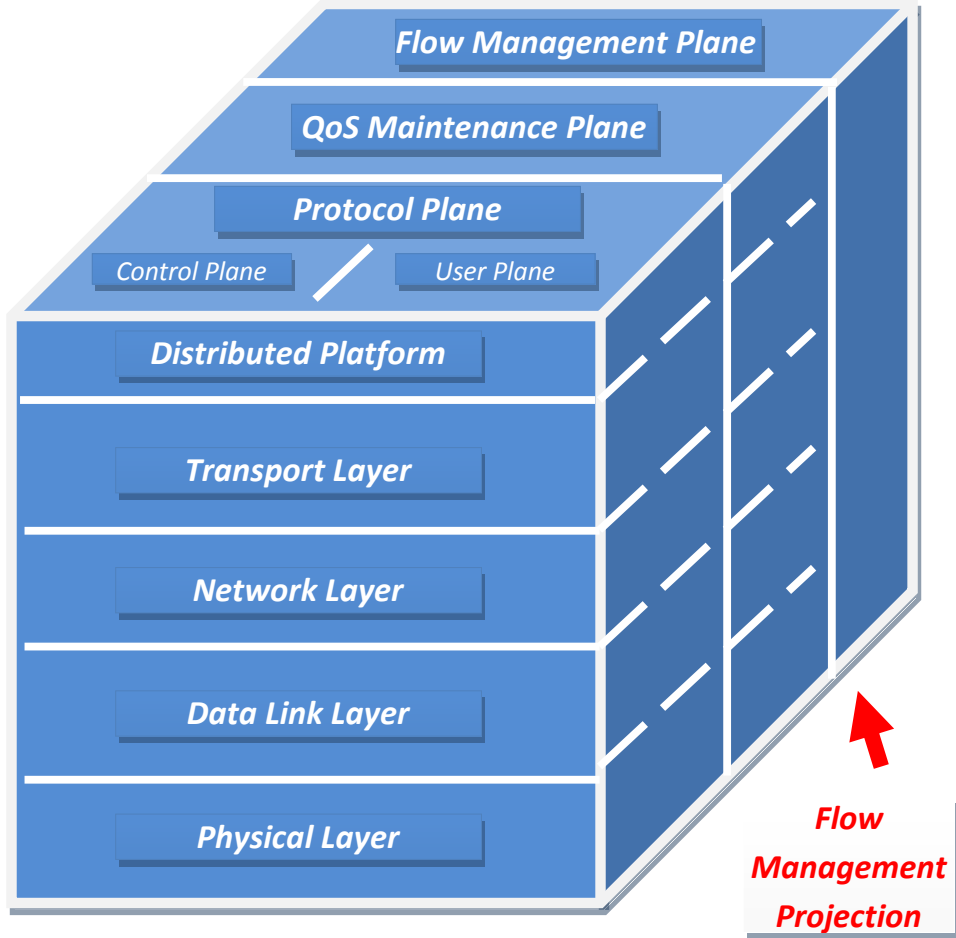

Fig. 1: Quality of Service Architecture section of Figure 1) illustrates the relationship between the three planes which work together to monitor and maintain end-to-end QoS.

\section{QOS MODEL CLASSIFICATION}

QoS models are classified into the following three major groups:

1. Integrated Services (IntServ) where framework provides explicit end-to-end reservations.

2. Differentiated Services (DiffServ) architecture which offers hop-by-hop differentiated treatment of packets.

3. Flexible QoS Model for MANETS (FQMM).

\subsection{Integrated services (IntServ)}

IntServ identifies three main categories of service concerning the integration: the traditionally best-effort services, real-time services and controlled link-sharing services.

Best-effort services are those we currently experience on the internet. They are characterized by absence of any QoS specifications. The network provides the quality that it actually can contribute. Examples of best-effort traffic are FTP, mail and FAX.

Real-time services are services that have very critical requirements in terms of end-to-end delay, probability of loss and bandwidth. They usually require a guarantee from the network.

Controlled link-sharing is a service that might be requested by network operators when they wish to share a specific link among a number of traffic classes. Network operators may set some sharing policies on the link utilization among these traffic classes; specifically some percentage of bandwidth may be assigned to each traffic class [4].

The IntServ QoS solution uses the resource reservation protocol (RSVP) to flood messages through the network, and reserves resources for every flow at every router hop from source to destination. Every router along the path must maintain soft states information. IntServ requires a lot of signaling; therefore the overhead is a concern when the network scale increases.

\section{Disadvantages}

IntServ has the following salient shortcomings in MANET environments:

Scalability: IntServ provides per-flow granularity, so the amount of state information increases proportionally with the number of flows. This results in a storage and processing overhead on routers, which is the well-known scalability problem of IntServ. The scalability problem is less likely to occur in current MANETs considering the small number of flows, the limited size of the network and the bandwidth of the wireless links. On the other hand, as the quality of wireless technology increases rapidly, high speed and large size MANETs may be a matter of fact some day.

Signaling: Signaling protocols generally contain three phases: connection establishment, connection maintenance and connection teardown. In highly dynamic networks such as MANETs this is no promising approach since routes may change very fast and the adaptation process of protocols using a complex handshaking mechanism would just be too slow. Furthermore the signaling overhead while maintaining the connection a potential problem as well.

\subsection{Differentiated services (DiffServ)}

DiffServ is a light weight alternative to IntServ. The concept of DiffServ is to differentiate the user data from control and management information. A field in the header of the Internet Protocol (IP) Data Unit was designed for these purposes: the Typeof-Service (TOS) field. The octet dedicated to this field indicates the specific treatment that the packet expects to receive from the network. The TOS bits are divided up as follows:

- 3 bits dedicated to priority of the datagram

- 3 bits define the type of service (TOS) which correspond to QoS expected by the IP datagram

- 2 bits are reserved for future use.

DiffServ does not maintain the state of each and every flow as IntServ does, but rather discriminates the packets according to their priority. The edge routers classify the traffic type, while the individual routers that forward the data will decide the fate of the packets according to local policies of the packet types. DiffServ is easier to maintain, more saleable and has less signaling than IntServ.

\section{Disadvantages}

The main drawbacks of a DiffServ approach in MANETs are listed below:

Soft QoS guarantees: DiffServ uses a relative-priority scheme to map the quality of service requirements to a service level. This aggregation results in a more scalable but also in more approximate service to user flow.

SLA (Service Level Agreement): DiffServ is based on the concept of SLA's. In the Internet an SLA is a kind of contract between a customer and its Internet Service Provider (ISP) that specifies the forwarding service the customer should receive. The Administration of a DiffServ domain must assure that sufficient resources are provisioned to support the SLA's committed by the domain [2]. Moreover, the DiffServ boundary nodes are required to monitor the arriving traffic for each service class and to perform 
traffic classification and conditioning to enforce the negotiated SLA's. In a completely ad hoc topology where there is no concept of service provider and client and where there are only clients it would be quite difficult to innovate QoS, since there is no obligation from somebody to somebody else what makes QoS almost infeasible.

Ambiguous core network: In MANETs though there is no clear definition of what is the core network because every node is a potential sender, receiver and router. This drawback would again take us back to the IntServ model where several separate flow states are maintained.

\subsection{Flexible QoS Model for Manet (FQMM)}

The idea of this model is to combine knowledge from the solutions offered in the wire-based networks and apply them to a new QoS Model. This model selectively uses the per-flow state property of IntServ, and the service differentiation of DiffServ. That is to say, for applications with high priority, per-flow QoS guarantees of IntServ are provided. On the other hand, applications with lower priorities are given per-class differentiation of DiffServ. This model is based on the assumption that not all packets in the network are actually seeking for highest priority, because then this model would result in a similar model with IntServ which have per flow provisioning for all packets. Therefore, FQMM applies a hybrid provisioning where both IntServ and DiffServ scheme are used separately.

The FQMM hybrid model defines three types of nodes, exactly as in DiffServ:

a) Ingress node: An ingress node which sends data.

b) Interior node: an interior node which forwards data to other nodes.

c) Egress node: an egress node which is the destination.

In FQMM model, a MANET represents one DiffServ domain where traffic is generated by applications running on an ingress node and terminating in an egress node.

\section{QOS TECHNOLOGIES}

\subsection{RSVP}

RSVP is a classic two-pass protocol using out-of-band signaling. The messages used are the Path message, which originates from the traffic sender, and the Resv message[6], which originates from the traffic receivers. The primary roles of the Path message are first to install reverse routing state in each router along the path, and second to provide receivers with information about the characteristics of the sender traffic and end-to-end path so that they can make appropriate reservation requests. Resv messages finally carry reservation requests to the routers along the distribution tree between receivers and senders. RSVP state is "soft-state", after a certain expire time, the state of the path and the reserved resource is released. Periodical issuing of Path or Resv messages are necessary to keep the reservation alive. Additional signaling information allows the soft state timeout to adapt to the refresh period. Furthermore, RSVP provides a routing triggered local repair [8] mechanism to overcome the need for a very fast refresh rate in order to react to route changes.

\section{Disadvantages}

There are many shortcomings of RSVP when used in MANETs: The two-pass reservation model employed by RSVP is not suitable for MANETs, especially in case of local repair.
RSVP is based on a fixed QoS level approach. As a consequence no mechanism for a fast adaptation to QoS changes can be provided. To solve this problem reservation requests should specify ranges of values instead.

Due to its out-of-band approach, RSVP produces a significant signaling overhead. This may be of importance if the refresh rate high because the message size is not negligible in RSVP. A high refresh rate might occur when no route-change notification service from the routing layer is available. This causes local repair to fail.

As an IntServ based protocol RSVPlacks of scalability. The amount of state information increases proportionally with the number of flows what causes storage and processing overhead. Although the scalability problem may not be likely to happen in current MANETs due to the limited size of the network and the bandwidth of wireless links, one could argue that it will occur with the development of fast radio technology and potential large number of users in the near future.

\subsection{INSIGNIA}

INSIGNIA is a signaling protocol designed explicitly for MANETs. It supports fast flow reservation, restoration and adaptation algorithms that are specifically designed to deliver adaptive realtime service. INSIGNIA implements an in-band approach by encapsulating some control signals in the IP option of every data packet, which is now called INSIGNIA option. Furthermore, flow state information is kept in every node of a particular path. This is done in a soft-state manner i.e., the flow state information is periodically refreshed by the received signaling information. In the following the basic operation of the signaling system is described with respect to INSIGNIA IP option.

INSIGNIA offers a one-pass reservation. When a source node wants to establish a reservation to a destination node it sets the reservation (RES) mode bit in the INSIGNIA IP option service mode of a data packet and sends the packet toward the destination. The bandwidth request field allows a source to specify its maximum (MAX) and minimum (MIN) bandwidth requirements. On reception of a RES intermediate routing nodes execute admission control to accept or deny the request. When a node accepts a request, resources are committed and subsequent packets are scheduled accordingly.

In contrast, if the reservation is denied, packets are treated as best effort (BE) mode packets. In the case where a RES packet is received and no resources have been allocated, the admission controller attempts to make a new reservation. This is a re-active local repair mechanism and commonly occurs when flows are rerouted during the lifetime of an ongoing session due to host mobility.

The bandwidth indicators field of INSIGNIA option plays an important role during reservation setup and adaptation. Reception of a setup request packet with the bandwidth indicator bit set to MAX indicates that all nodes encountered have sufficient resource to support the maximum bandwidth requested. On the other hand, a bandwidth indicator set to MIN implies that at least one of the intermediate nodes between the source and destination is a bottleneck node and the maximum bandwidth requirement may not be met.

When a reservation is received at the destination node, INSIGNIA checks the reservation establishment status. The status is determined by inspecting the IP option field service mode, which should be set to RES. If the bandwidth indication is set to MAX, this implies that all nodes between a source-destination pair have successfully allocated resources to meet the QoS requirements of the source node. In contrast if the bandwidth indication is set to MIN this indicates that only the minimum bandwidth can be 
currently supported. As a result "partial reservations" will exist between source and bottleneck node, these resources remain reserved until explicitly released. QoS reporting message can be sent by destination nodes to inform source nodes of the ongoing status of flows. They do not have to travel on the reverse path toward a node.

The INSIGNIA system supports two QoS report commands in order to provide some kind of adaptation.

A scale-down command requests a source either to send with the rate specified as MINIMUM instead of MAXIMUM or to send its packets as best effort instead of MINIMUM depending on the current sending rate of the source node. This will have the effect of clearing any partial reservation.

A scale up requests a source node to initiate a reservation for some MINIMUM or MAXIMUM rate, depending on the actual flow state.

\section{Disadvantages}

Although INSIGNIA presents a quite promising approach to QoS support in ad hoc networks, the system still lacks of some basic mechanisms. The most frequently mentioned drawback of INSIGNIA in literature is its scalability problem due to the flow state information which is kept within the nodes of a certain path. This is an inherent problem of IntServ but it is doubtful whether it will be of importance for MANETs in future.

INSIGNIA's bandwidth usage is not efficient. The extra reservation on the path from the sending node to the bottleneck is a waste of bandwidth until an explicit release message is sent. Although this waste won't last long, topology changing of MANET will make this reservation waste propagate frequently. Furthermore releasing partial reservations using QoS reports enforces source nodes either to set the bandwidth indicator of the INSIGNIA option field to MINIMUM or to send the packets as best effort depending on the actual flow state. In both cases the opportunity to scale up is lost.

INSIGNIA does not provide any mechanism to dynamically change the frequency by which control signals are inserted into the data packets. This imposes a major processing overhead on the network.

Only two bandwidth levels to be used are offered, MINIMUM and MAXIMUM. A more fine-grained approach would be needed in order to satisfy application requirements and to fully exploit the resources available.

\subsection{IMAQ}

iMAQ[4] is a cross-layer architecture to support the transmission of multimedia data over a MANET. They use a location-based proactive QoS-Routing. Neither hard QoS guarantees can be provided nor are any resources reserved. Because cross-layer designs and QoSRouting are not within the scope of this document, the iMAQ approach is not considered any further.

\subsection{INORA}

INORA [5] is a QoS support mechanism that makes use of the INSIGNIA in-band signaling and TORA routing protocol for MANETs. INORA represents a QoS-signaling approach in a loosely coupled kind of manner. The idea is based upon the property of TORA to provide multiple routes between a given source and destination. While INSIGNIA does not take any help from the network with regard to redirecting the flow along routes which are able to provide the required QoS guarantees, INORA gives feedback to the routing protocol on a per-hop basis to direct the flow along the route that is able to satisfy the QoS requirements of the flow.
Beyond doubt the concept of 'loosely coupling' QoS-signaling and routing is a very promising approach and the shortcomings of INORA mostly are the shortcomings of INSIGNIA. However, the interface for signaling to access routing should be as generic as possible in order to guarantee portability.

\section{CONCLUSION}

This paper tried to provide a brief overview on the Quality of Service model, architecture issues and solution given in the wirebased IP network, where much more progress has been done and Signaling, Routing protocols cannot be directly mapped to MANETS, because of the bandwidth constrains and dynamic topology of such networks. Also discussed about the FQMM model for MANET, but several issues are still under study and new models are being developed to solve issues such as decision upon traffic classification, allotment of per flow or aggregated service for the given flow, amount of traffic belonging to per flow service. A great deal of work remains to be done in this area until it reaches the human society in an easy form.

\section{REFERENCES}

[1] Campbell A., Coulson G., Garc.A. F., and Hutchison D., August 1992, A Continuous Media Transport and Orchestration Service", Proc. ACM SIGCOMM Ô92, Baltimore, Maryland, USA.

[2] Campbell A., Coulson G., Garc.A F., Hutchison D., and Leopold H. 1993, Integrated Quality of Service for Multimedia Communications, Proc. IEEE INFOCOMÕ93, pp. 732-739, San Francisco, USA.

[3] Garc A., F., A Continuous Media Transport and Orchestration Service, 1993, PhD Thesis, Department of Computing, Lancaster University, Lancaster LA1 4YR, UK.

[4] imaq: An integrated mobile ad hoc qos framework. http://cairo.cs.uiuc.edu/adhoc/.

[5] Dharmaraju D., Roy-Chowdhury A., Hovareshti P., John S. Baras, , 2002, Inora - a unified signaling and routing mechanisms for qos support in mobile ad hoc networks, Proceedings of the International Conference on Parallel Processing Workshops.

[6] Chen S., Nahrstedt K., 1998, An Overview of Quality-ofService Routing for the next Generation High -Speed Networks: Problems and Solutions, IEEE Network Magazine, vol.12, pp. $64-79$.

[7] Lee S.B. and Campbell A.T., 1998, INSIGNIA: In- band signaling support for QoS in mobile ad hoc networks." KL In 5th Int. Workshop on Mobile Mul- timedia Comm. (MoMuc'98), Berlin, Germany.

[8] RFC2386.

Mukesh Kumar received the B.Tech .degree in Computer Science \& Engineering from M. M. College of Engineering, Mullana ( Kurushetra University, Kurushetra) and M.Tech in Computer Science and Engineering from Guru Jambeshwer University of Science \& Technology Hisar in 2001 and 2004 respectively. He is currently doing his Ph.D degree from The Technological Institute of Textile and Science, Bhiwani-127021, (Under M. D. University Rothak) Haryana - India. Currently he is a Ph.D Student in the department of computer Engineering, The Technological Institute of Textile and Science, Bhiwani-127021. His area of interest is Adhoc Networks, Computer Networks, Artificial Intelligence and Network security. He has published articles in International Journals and presented many papers in national and International Conferences. 